Muscle fiber regeneration in long term denervation

Eur J Transl Myol - Basic Appl Myol 2015; 25 (2): 77-92

\title{
Persistent muscle fiber regeneration in long term denervation. Past, present, future
}

Ugo Carraro (1), Simona Boncompagni (2), Valerio Gobbo (3), Katia Rossini (4,5), Sandra Zampieri (4,5), Simone Mosole (4,5), Barbara Ravara (4,5), Alessandra Nori (4), Roberto Stramare (6), Francesco Ambrosio (7), Francesco Piccione (1), Stefano Masiero (8), Vincenzo Vindigni (9), Paolo Gargiulo (10), Feliciano Protasi (2), Helmut Kern (5,11), Amber Pond (12), Andrea Marcante (1)

(1) Department of Neurorehabilitation, Foundation San Camillo Hospital, I.R.C.C.S., Venice, Italy; (2) CeSI, Center for Research on Aging, Department of Neuroscience, Imaging and Clinical Sciences, University G. d'Annunzio of Chieti, Italy; (3) C.N.R. Institute of Neuroscience, Department of Biomedical Science, University of Padova, Italy; (4) Translational Myology, Interdepartmental Research Center of Myology of the University of Padova (CIR-Myo), Department of Biomedical Science, Padova, Italy; (5) Ludwig Boltzmann Institute of Electrical Stimulation and Physical Rehabilitation, Vienna, Austria; (6) CIR-Myo, Department of Medicine, Radiology Unit, University of Padova, Italy; (7) Antalgic Laboratory, Department of Medicine, University of Padova, Italy; (8) CIR-Myo, Department of Neuroscience, Rehabilitation Unit, University of Padova, Italy; (9) CIR-Myo, Department of Neuroscience, Plastic Surgery Unit, University of Padova, Italy; (10) Department of Science, Education, Innovation, Landspitali University Hospital, Reykjavik, Iceland; (11) Department of Physical Medicine, Wilhelminenspital, Vienna, Austria; (12) Anatomy Department, Southern Illinois University, School of Medicine, Carbondale, Illinois, USA

\begin{abstract}
Despite the ravages of long term denervation there is structural and ultrastructural evidence for survival of muscle fibers in mammals, with some fibers surviving at least ten months in rodents and 3-6 years in humans. Further, in rodents there is evidence that muscle fibers may regenerate even after repeated damage in the absence of the nerve, and that this potential is maintained for several months after denervation. While in animal models permanently denervated muscle sooner or later loses the ability to contract, the muscles may maintain their size and ability to function if electrically stimulated soon after denervation. Whether in mammals, humans included, this is a result of persistent de novo formation of muscle fibers is an open issue we would like to explore in this review. During the past decade, we have studied muscle biopsies from the quadriceps muscle of Spinal Cord Injury (SCI) patients suffering with Conus and Cauda Equina syndrome, a condition that fully and irreversibly disconnects skeletal muscle fibers from their damaged innervating motor neurons. We have demonstrated that human denervated muscle fibers survive years of denervation and can be rescued from severe atrophy by home-based Functional Electrical Stimulation (h-bFES). Using immunohistochemistry with both non-stimulated and the h-bFES stimulated human muscle biopsies, we have observed the persistent presence of muscle fibers which are positive to labeling by an antibody which specifically recognizes the embryonic myosin heavy chain (MHCemb). Relative to the total number of fibers present, only a small percentage of these MHCemb positive fibers are detected, suggesting that they are regenerating muscle fibers and not pre-existing myofibers re-expressing embryonic isoforms. Although embryonic isoforms of acetylcholine receptors are known to be re-expressed and to spread from the end-plate to the sarcolemma of muscle fibers in early phases of muscle denervation, we suggest that the MHCemb positive muscle fibers we observe result from the activation, proliferation and fusion of satellite cells, the myogenic precursors present under the basal lamina of the muscle fibers. Using morphological features and molecular biomarkers, we show that severely atrophic muscle fibers, with a peculiar cluster reorganization of myonuclei, are present in rodent muscle
\end{abstract}




\section{Muscle fiber regeneration in long term denervation}

Eur J Transl Myol - Basic Appl Myol 2015; 25 (2): 77-92

seven-months after neurectomy and in human muscles 30-months after complete Conus-Cauda Equina Syndrome and that these are structurally distinct from early myotubes. Beyond reviewing evidence from rodent and human studies, we add some ultrastructural evidence of muscle fiber regeneration in long-term denervated human muscles and discuss the options to substantially increase the regenerative potential of severely denervated human muscles not having been treated with h-bFES. Some of the mandatory procedures, are ready to be translated from animal experiments to clinical studies to meet the needs of persons with longterm irreversible muscle denervation. An European Project, the trial Rise4EU (Rise for You, a personalized treatment for recovery of function of denervated muscle in long-term stable SCI) will hopefully follow

Key Words: Human muscle, Conus-Cauda Equina syndrome, Spinal cord injury, Permanent muscle denervation, Severe atrophy and nuclear clumpings, Muscle fiber regeneration, Homebased functional electrical stimulation (h-b FES), Recovery of tetanic contractility, Myogenic stem cells

Eur J Transl Myol - Basic Appl Myol 2015; 25 (2): 77-92

Skeletal muscle undergoes a rapid loss of both mass and contractile force in response to loss of neural input such as occurs in cases of sciatectomy in rats and with spinal cord injury (SCI) in humans. The atrophy subsequent to SCI is especially severe when the lesion involves lower motor neurons (LMN) because, if denervation is irreverisble, the muscle tissue ultimately undergoes both fibrosis and fat substitution, thus producing denervated degenerated muscle (DDM). Unfortunately, long-term permanent denervation of muscle tissue is an under-studied pathologic condition. This situation may be attributable to the general belief that muscle fibers will eventually disappear after weeks or months of disconnection from the nervous system and its provision of trophic factors (e.g., those related to acetylcholine, agrin, BDNF and other as yet unknown chemical factors) released from axonal

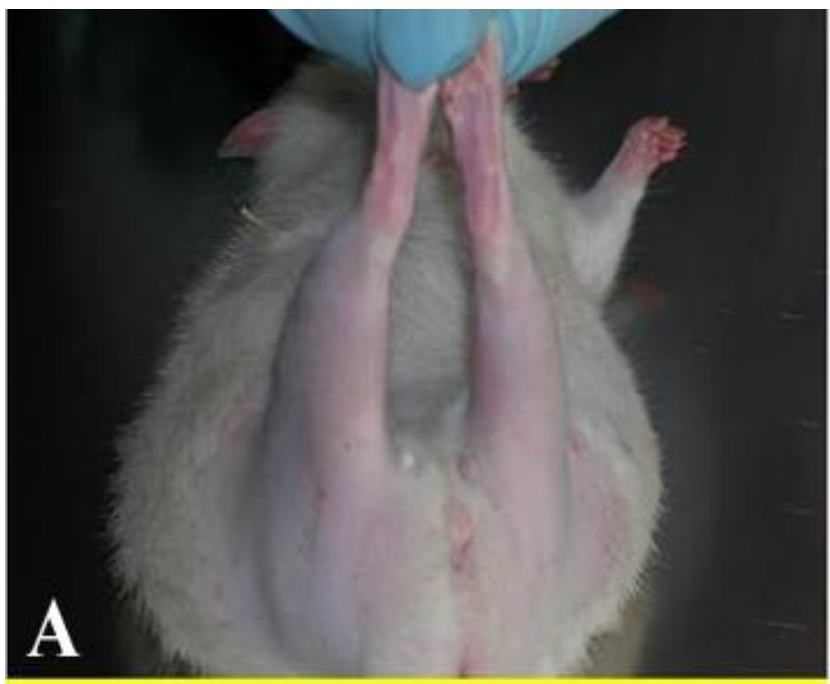

Normal Adult Rat endings. ${ }^{1-3}$ Because both the response of rat muscle to permanent denervation (Figure 1) and the response of human muscle to SCI is an extreme loss of muscle mass, there has been a good deal of skepticism aimed at the efficacy of our studies of home-based Functional Electrical Stimulation (h-bFES) as a potential therapy to improve structure, appearance and tetanic contractility of permanently denervated human muscles. ${ }^{4}$ Based upon the fact that at late stages of denervation severely atrophic skeletal muscle does not respond to electrical stimulation, many neurologists believe that muscle degeneration is irreversible and thus therapy is not merited. Here, we respond to such skepticism by discussing evidence to support the value of our technique, namely the facts that: 1) myofibers are indeed present in rat muscle one year after denervation $;^{5}$ 2) atrophied denervated human muscle maintains surviving and regenerating myofibers over

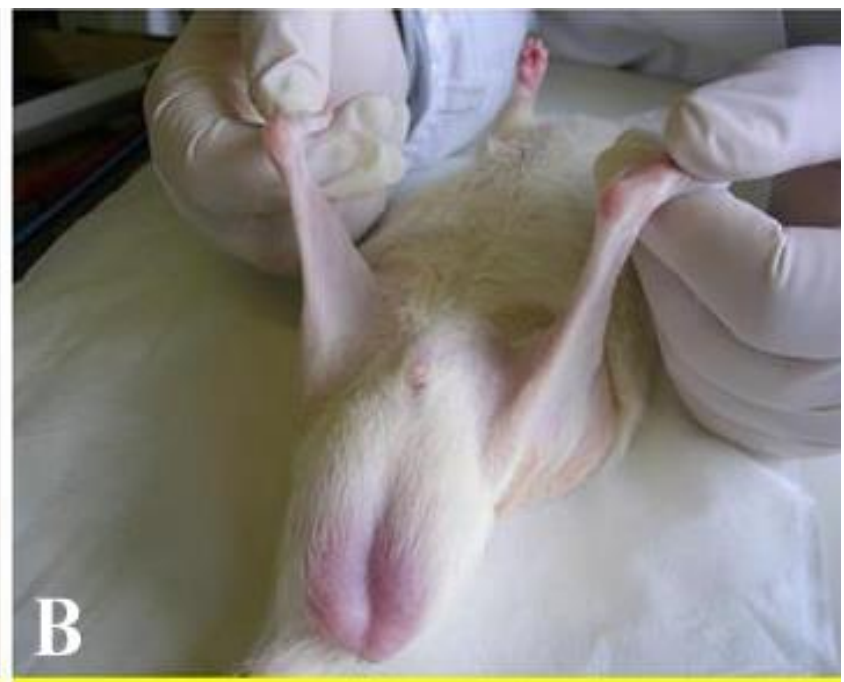

9-month Bilateral Sciatectomy

Fig 1. Macroscopic aspects of rat posterior legs. In comparison to adult normal rat (A), the 9-month denervated legs (B) appear to have completely lost their muscle mass. 


\section{Muscle fiber regeneration in long term denervation}

Eur J Transl Myol - Basic Appl Myol 2015; 25 (2): 77-92
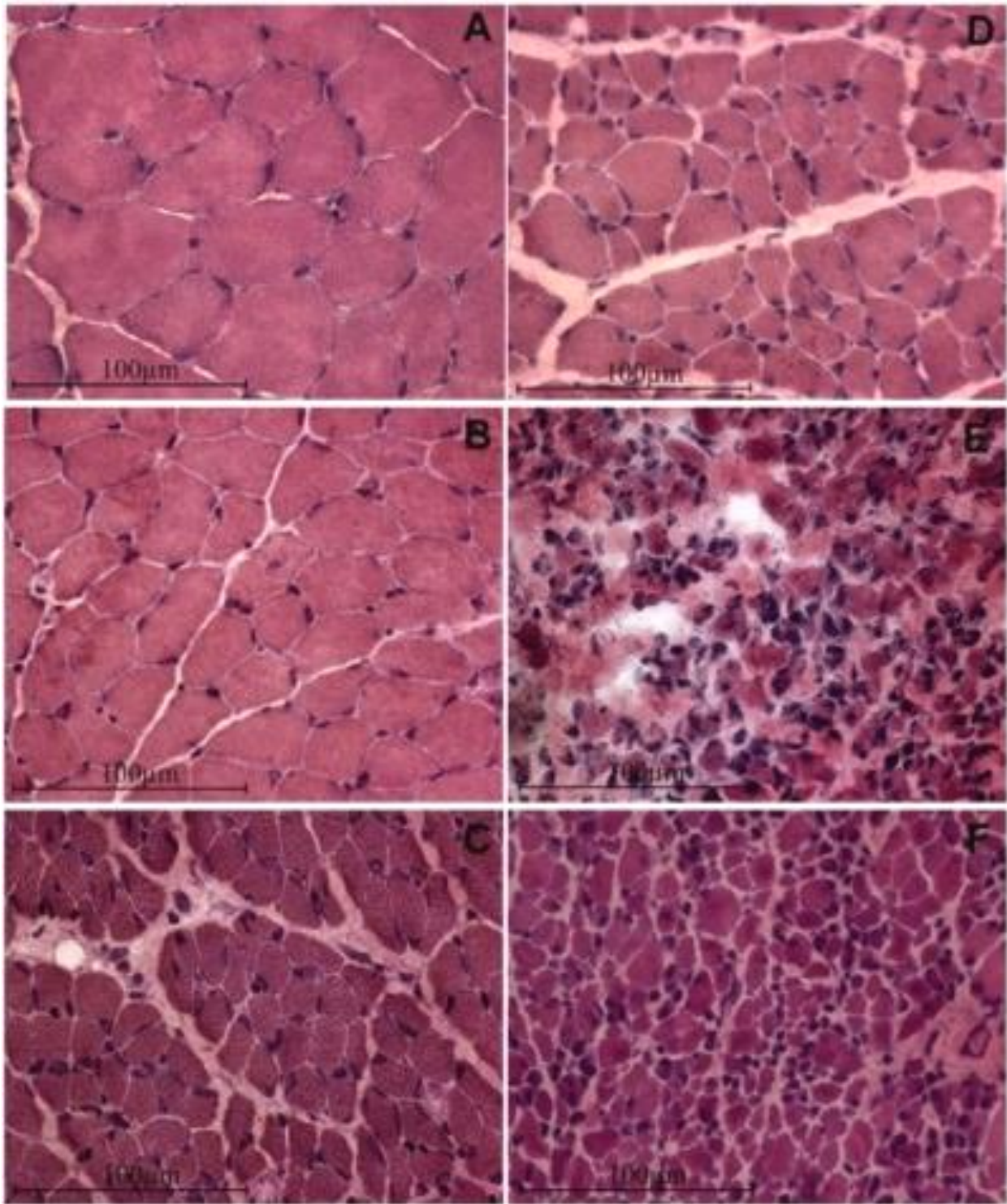

Fig 2. Long term denervation atrophy of the TA muscle in bilaterally sciatectomized rats. H-E stain. A, Normal muscle; B, 1-month, C, 2-month, D, 4-month; E, 8-month; F, 12-month post denervation. Innervated TA muscles are characterized by large, well packed myofiber profiles and minimal intermyofiber spaces. The first stage of TA denervation is characterized by progressive reduction in muscle fiber diameter. In the middenervation stage there is a further reduction up to 4-months denervation, in which muscle fiber size is about $10 \%$ of normal. Then, the fiber size does not change significantly at least up to 1-year after denervation by bilateral sciatectomy.

time; ${ }^{4}$ 3) immunochemical evidence of embryonic myosin and evidence of biomarkers of myogenic processes in rodents suggest that myogenesis may occur in denervated muscles; and 4) h-bFES improves ultrastructure, macro-structure, mass and contractility of permanently denervated human muscle. Indeed, denervated human muscle tissue responds to h-bFES treatment by recovering surviving myofibers and, possibly, also those recently regenerated muscle fibers which are more responsive to electrical stimulation., ${ }^{3,4}$
Long term survival of permanently denervated muscle fibers in mammals

In support of the possibility of muscle recovery, first, we argue that muscle fibers are recognizable, even at the light microscope level, in the Tibialis Anterior (TA) muscle of sciatectomized rats for up to 1-year post denervation (Fig. 2). ${ }^{5}$ Addtionally, we point out that electron microscopic analyses of the tissue remaining in long term denervated rat leg muscles ${ }^{6,7}$ show that the deterioration which occurs in response to denervation is ameliorated by electrical stimulation, 
Muscle fiber regeneration in long term denervation

Eur J Transl Myol - Basic Appl Myol 2015; 25 (2): 77-92

\section{Aneurally regenerated muscle}
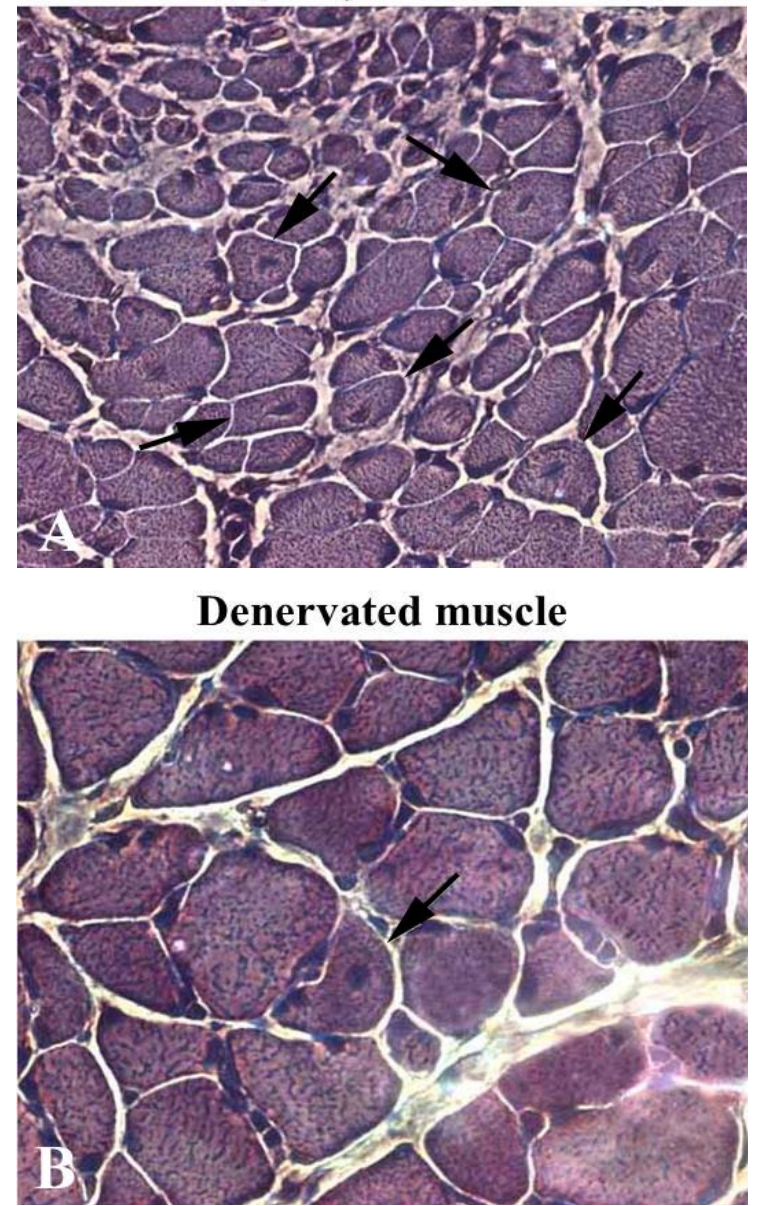

Fig 3. Semithin sections, toluidine blue stain. Note in A the gradient of size of muscle fibers and the presence of central nuclei in myofibers of denervated and regenerated rat soleus muscle 30 days after bilateral sciatectomy and unilateral marcaine-induced muscle damage. Spontaneous regeneration is also present in 30 days denervated contralateral muscle as suggested by the central nucleus in one myofiber (arrow in B). 40x.

supporting our belief that long-term permanently denervated muscle may be restored structurally (and eventually to functional behavior) in particular if this anti-atrophy/recovery process (i.e., h-bFES) is started before muscular atrophy becomes severe. ${ }^{3,4}$ Rodent models of muscle denervation, in particular after free muscle transplantation, ${ }^{2}$ have demonstrated that lasting periods ( 1 to 6 months post denervation) of myofiber "atrophy" elapse before the detection of muscle degeneration and also report the formation of very small myofibers that may constitute up to $25 \%$ of the total muscle fibers in denervated leg muscles of rats. ${ }^{6,7}$ Further, Borisov et al., ${ }^{6}$ thoroughly characterized severely atrophic rat muscle fibers at the ultrastructural
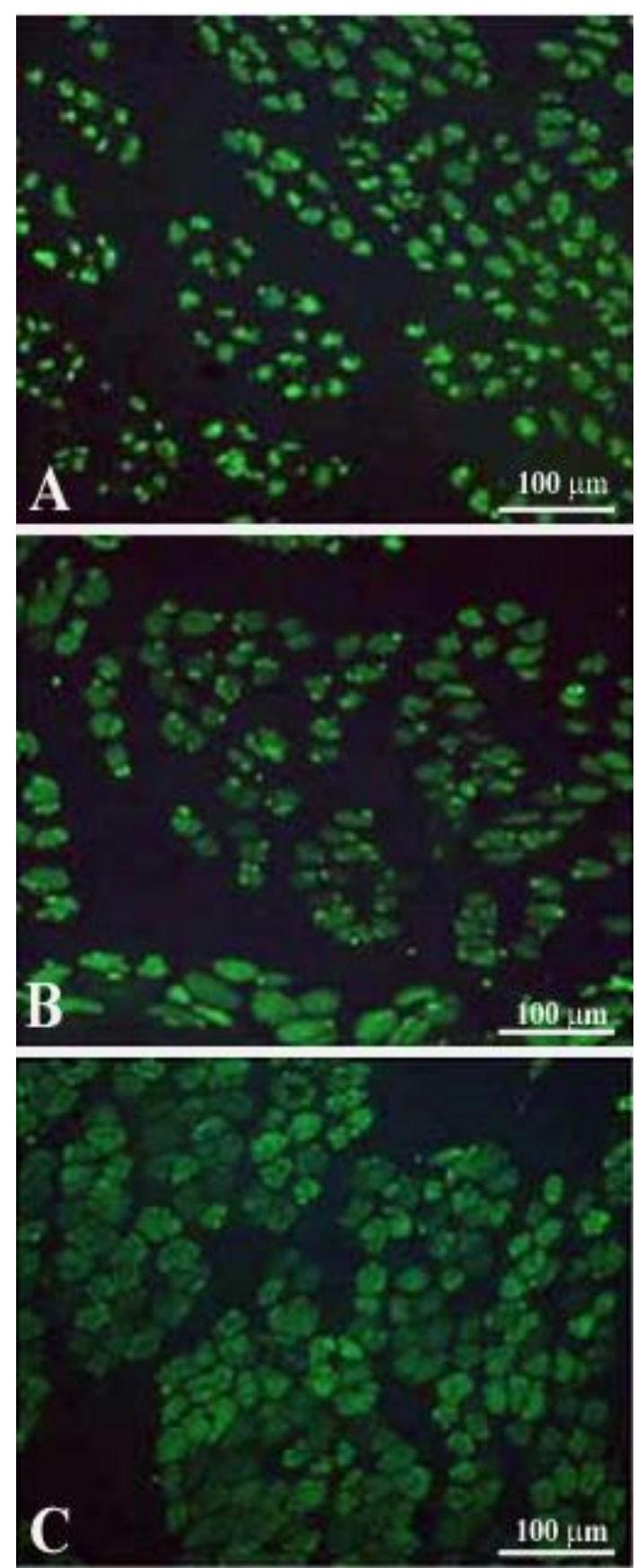

Fig 4. Marcaine induced muscle fiber damage and regeneration in denervated rat muscle, after bilateral sciatectomy and unilateral marcaine-induced muscle damage. Time course of MHCemb expression in aneurally regenerated rat muscle. A, 5 days, B, 7 days, $C, 10$ days after Marcaine muscle damage. Note that the muscle fibers increase in size during the first two weeks, but that the signal begins to fade at ten days of muscle regeneration 


\section{Muscle fiber regeneration in long term denervation}

Eur J Transl Myol - Basic Appl Myol 2015; 25 (2): 77-92

Aneurally regenerated muscle

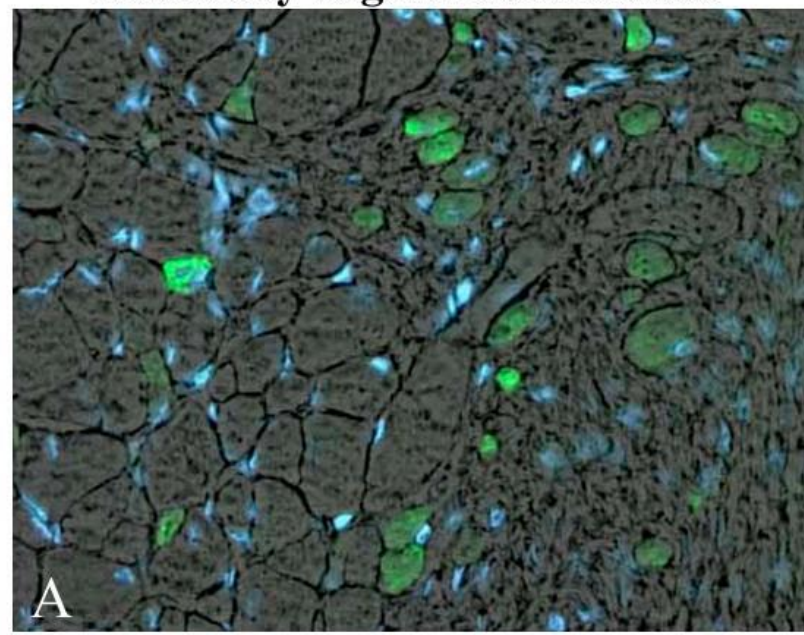

\section{Denervated muscle}

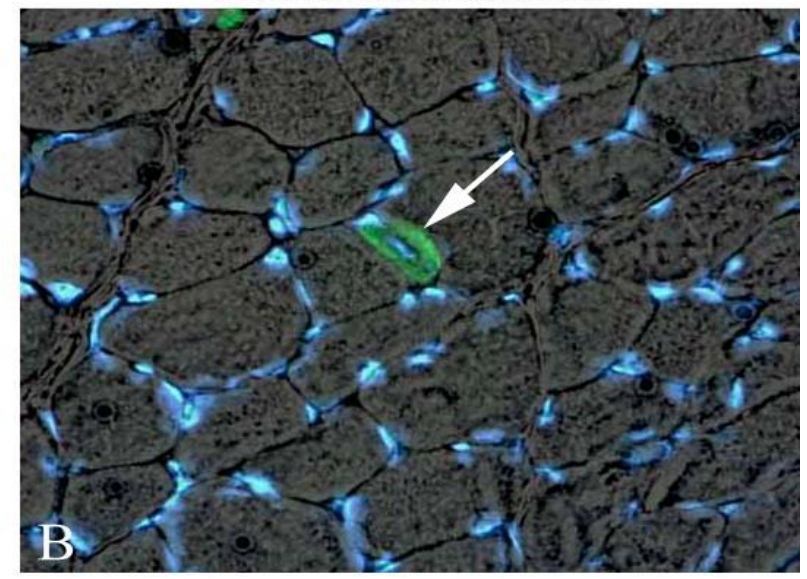

Fig 5. Marcaine induced muscle fiber regene-ration in denervated rat soleus 30 days after bilateral sciatectomy and unilateral marcaine-induced muscle damage. Double exposure anti MHC emb (green)/Hoechst (blue staining of nuclei). A, The 30 days aneurally regenerated muscle shows only some myofibers positive for MHCemb, while the majority is negative. B, 30 days contralateral denervated muscle presents only one MHCemb positive myofiber with central nucleus (regenerated muscle fiber). Note that all other myofibers are negative. Magnification, 40x.

level and revealed that they contain a contractile system, although poorly developed and with many degenerated elements. The authors suggested that these "incompletely differentiated" muscle fibers arise from satellite cells that have detached from their parent myofibers and may represent an "abortive" attempt at myogenesis. ${ }^{6}$ Schmalbruch and colleagues have described similar myofibers in rat muscle denervated for 6-10 months. ${ }^{7}$ The majority of these muscle cells were so small, down to $1 \mu \mathrm{m}$ in diameter, that they could not be identified by routine light microscopy. Schmalbruch and colleagues further noted that some of the smaller fibers were completely devoid of myofilaments. Accordingly, they concluded that these fibers are the result of repeated cycles of necrosis and regeneration occurring in the chronically denervated skeletal muscle, based on their reporting of unequivocally necrotic muscle fibers. ${ }^{7}$ Carraro and colleagues found similar featurers in rat hemidiaphragm denervated for $12-16$ months. ${ }^{8}$

Taken together, the above examples provide morphological evidence that very small myofibers may not only represent extreme examples of severely atrophied muscle fibers surviving long-term denervation (see below), but may also occur as the result of myofiber regeneration followed by atrophy in chronic denervation. ${ }^{6}$ Indeed, Mussini et al. ${ }^{9}$ have shown that marcaine damage of permanently denervated rat muscles produces unequivocal morphological features of muscle damage and regeneration and that repeated marcaine infusion of the aneural regenerated rat muscle was followed by a second round of muscle damage and regeneration, demonstrating that the regenerative process is accompained by survival and/or proliferation of myogenic cells with structural and functional characteristics of satellite cells (or, in other words, "stem cells"). ${ }^{9}$ Conclusive evidence of aneural satellite cell activation, proliferation, fusion and progression to small muscle fibers was obtained by gel electrophoresis which revealed the transient presence of the embryonic isoforms of the myosin light and heavy chains (MLCemb, MHCemb, respectively) in regenerating rat muscles, ${ }^{10}$ and by immunolabeling aneurally regenerating muscle cryosections (Fig. 3) with an antibody that recognizes epitopes of the MHCemb (Figs. 4 and 5). ${ }^{11,12}$ Note that in Fig. 4 the size of the regenerated muscle fibers increases up to 10 days (Fig. 4, A-C), at least, but that the immunostain starts to fade in several muscle fibers at this time of regeneration (Fig. 4, C). At 30 days of regeneration in aneural muscles (Fig. 3, A), indeed, the majority of the muscle fibers are negative for MHCemb protein and only some small myofibers are positive (Fig. 5, A). It is interesting to note that in rat muscle denervated for 30 days (Fig. 3, B) only one small round muscle fiber with a central nucleus is stained (Fig. 5, B) with the antiMHCemb antibody that we are using for our studies in rodents and human muscles, ${ }^{13}$ while all other larger denervated muscle cells, with subsarcolemmal nuclei, are negative (Fig. 5, B). In our opinion, the anti-body we are using recognizes epitopes of the embryonic myosin heavy chains that are not re-expressed in adult muscle fibers during the first month of denervation, at least in the rat muscles. ${ }^{13}$ The same result was observed when the marcaine damage and the myogenic response 


\section{Muscle fiber regeneration in long term denervation}

Eur J Transl Myol - Basic Appl Myol 2015; 25 (2): 77-92

occurred in rat muscles permanently denervated for 4 to 6 months. ${ }^{13}$ Further evidence of muscle fiber regeneration is the detection of myogenin and MRF4 gene expression in response to long-term muscle denervation, ${ }^{14,15}$ in spite of the down-regulation of activity-dependent genes in long-term denervated rat muscle. ${ }^{15}$ In conclusion, we are confident that expression of the MHCemb is the result of a cellular process of muscle regeneration.

It is also worth noting that the long term denervated muscle fibers of rats are able to retain a functional excitation-contraction coupling (ECC) apparatus that responds in vitro to electrical stimulation for several months, despite lost contractility. ${ }^{16}$ This provides a potential mechanism for the muscle recovery that occurs in response to rehabilitation strategies developed on the basis of empirical clinical observations. ${ }^{16}$ To define the time course and potential effects of electrical stimulation on permanently denervated muscle, we evaluated excitation-contraction coupling of rat leg muscles during their progressive response to long-term denervation ${ }^{16}$ by determining: 1) ultrastructural analysis; 2) specific binding to dihydropyridine receptors, ryanodine receptors, $\mathrm{Ca}^{++}$ channels and extrusion $\mathrm{Ca}^{++}$pumps; 3) gene transcription and translation of $\mathrm{Ca}^{++}$-handling proteins; and 4) in vitro mechanical properties and electrophysiological analyses of sarcolemmal passive properties and L-type $\mathrm{Ca}^{++}$current parameters. We found that in response to long-term denervation: 1) isolated muscle that is unable to twitch in vitro by electrical stimulation has very small myofibers but may show a slow caffeine contracture; 2) only roughly half of the muscle fibers with "voltage-dependent $\mathrm{Ca}^{++}$ channel activity" are able to contract; 3) the ECC mechanisms are still present and, in part, functional; 4) ECC-related gene expression is upregulated; and 5) at any time point, there are muscle fibers that are more resistant than others to denervation atrophy and disorganization of the ECC apparatus. ${ }^{16}$ These results support the hypothesis that prolonged "resting" $\left[\mathrm{Ca}^{++}\right] \mathrm{i}$ may drive progression of muscle atrophy to degeneration and that electrical stimulation-induced $\left[\mathrm{Ca}^{++}\right] \mathrm{i}$ modulation may mimic the lost nerve influence, playing a key role in modifying the gene expression of denervated muscle. ${ }^{16}$ Hence, these data provide a potential molecular explanation for the muscle recovery that occurs in response to rehabilitation strategies developed on the basis of empirical clinical observations. $^{4}$

\section{Severe atrophy and nuclear clumps mark human muscle fibers surviving long-term denervation (2 to 6 years after SCI)}

In rodents, three to seven months after denervation, muscle fibers undergo a stage of slow progressive atrophy that results in a consistent reduction (up to $90 \%$ ) of the muscle volume. At this late stage, the denervated muscle still contains numerous severely atrophic muscle fibers, some of which have lost all the contractile proteins and the spiral distribution of myonuclei that have aggregated in the center of the muscle fiber (nuclear regroupings or clumps). At the same time, adipocytes and collagen sheets fill some of the empty spaces of the muscle tissue and finally fibrosis prevails. ${ }^{1,5,13,17}$

The permanent injury of LMNs in humans causes rapid denervation atrophy of skeletal muscle fibers that mainly occurs during the first few weeks post trauma. In biopsies of quadriceps muscle harvested from humans who have experienced complete LMN lesion (complete Conus-Cauda Equina Syndrome), we have observed events similar to those occurring in rats, but over a more extended period of time (1 to 6 years). Mild atrophy (that corresponds to an approximate $50 \%$ decrease in muscle fiber size) appears within a few weeks. ${ }^{18}$ It progresses during the first two years of denervation, while severe atrophy (a stage in which muscle fiber size decreases to $10 \%$ of normal values) appears after two to three years, accompanied by a progressive degeneration of the muscle tissue. ${ }^{18,19}$ This muscle time-course is not unique to humans, but is also a common feature in mammals larger than rodents (rabbits, guinea pigs, sheep). ${ }^{20-22}$ After the second year of persistent LMN denervation, human muscle tissue is progressively enriched with severely atrophic fibers which are depleted of myofibrillar apparati and, in longitudinal section, present with aggregations of tens of nuclei (nuclear clumps) separated by stretches of empty myoplasm. In transverse sections, these severely atrophic muscle fibers show three or more centrally located nuclei and typically no contractile structures. ${ }^{1}$ We analyzed the time-course of events in severely atrophic human muscle fibers presenting with nuclear clumps in complete LMN denervated quadriceps muscle biopsies harvested from one to ten years after lumbar-ischiatic SCI. ${ }^{23}$ We noted that nuclear clumps are common in the severely atrophic muscle fibers of LMN denervated human muscle from three to six years post injury (Fig. $6 \mathrm{~A}$ ). Specifically, in the muscle sections from biopsies harvested from one to two years after complete denervation, we observed myonuclear clumps in $2 \pm 5 \%$ of fibres (mean $\pm \mathrm{SD}$ ). In biopsies harvested three to five years after SCI, the percentage of fibres with myonuclear clumps abruptly and significantly increased to $27 \pm 9 \%$ ( $1<<0.001$ ); however, the value significantly decreased in biopsies harvested more than six years from SCI to $4 \pm 4 \%$ ( $<<$ 0.001 vs three-six years of denervation).

Fig. 6, B and Fig. 7 show cryosections from 4.0-year and 5.4-year LMN denervated human muscle biopsies, respectively, after staining (in green) with an antiMHCslow antibody. In Fig. 7 the white arrows point to slow-type (green) atrophic muscle fibers with centralized nuclei labeled in blue by Hoechst 33258 . The black arrowheads indicate two less atrophic fast 
Muscle fiber regeneration in long term denervation

Eur J Transl Myol - Basic Appl Myol 2015; 25 (2): 77-92
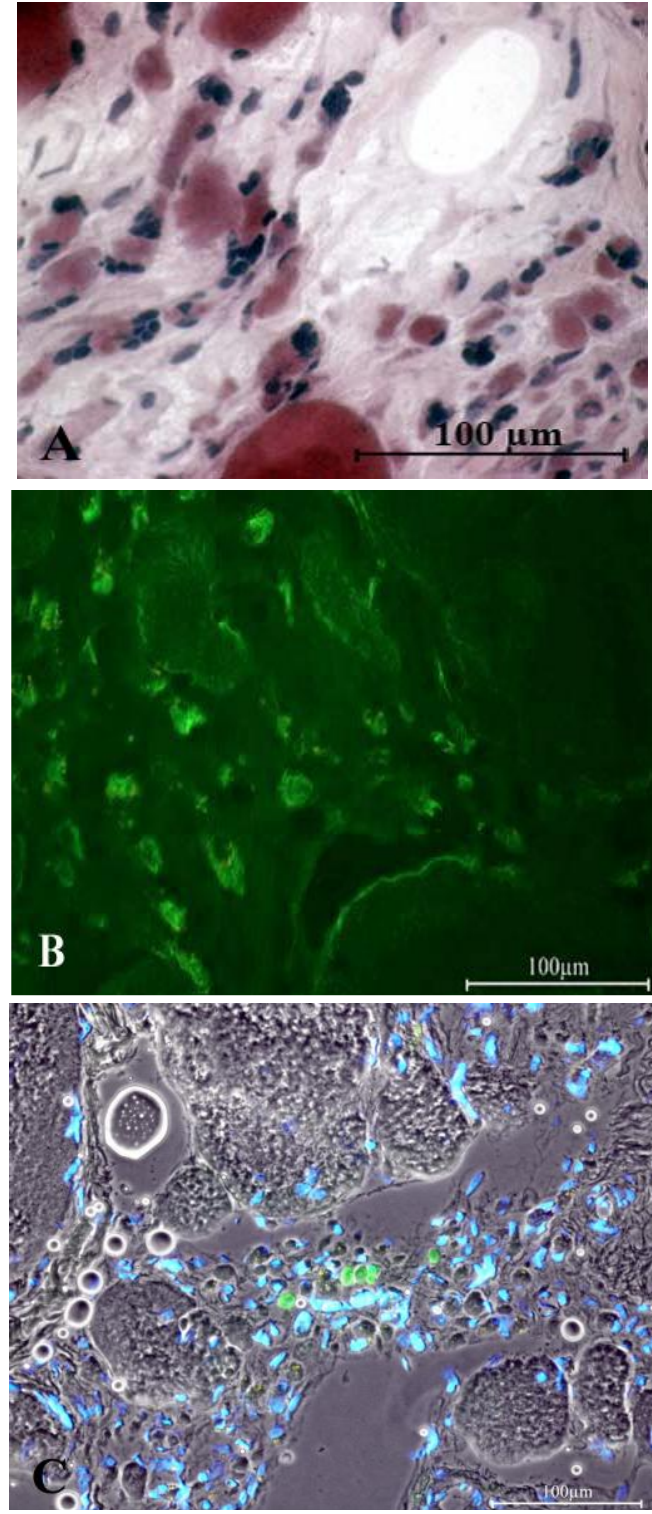

Fig 6. . A, Four-year $L M N$ denervated quadriceps muscle. H\&E stain. Some of the severely atrophic myofibers have remnants of the contractile machinery, while others are empty of any sarcomeric structures.

$B$, Immunohistochemistry of the 4 years LMN denervated human muscle. Several atrophic slow type myofibers are stained in green by the anti-MHCslow antibody.

$C, 4$ years $L M N$ denervated human muscle. A few muscle fibers are stained in green by the anti-MHCemb. Nuclei are stained by Hoechst 33258. Scale bar: $100 \mu \mathrm{m}$.

type muscle fibers (not stained by the anti-MHCslow antibody), which contain several centralized nuclei. The immunohistochemical analyses presented in panel B of Fig. 6 and in Fig 7 show that both fast and slow muscle fibers undergo severe atrophy and nuclear

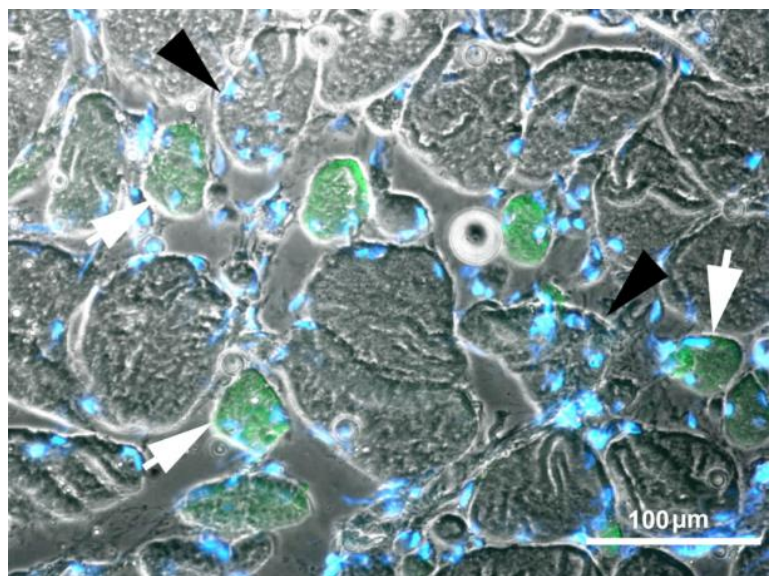

Fig 7. Four-year LMN human denervated quadriceps muscle. Immunohistochemical staining with anti-MHCslow shows that both the greenlabeled slow type muscle fibers (white arrows) and the fast (larger, not stained muscle fibers noted by black arrowheads) present several central nuclei. Scale bar: $100 \mu \mathrm{m}$.

relocation and that this relocation of nuclei precedes the disappearance of all the contractile proteins. On the other hand, only a few ( $1 \%$ of the muscle fibers we were able to count) small muscle fibers from the longer term denervated muscles stain in green with an antiMHCemb anti-body (Fig 7, C). These morphological aspects suggest that severe atrophy with consequent nuclear redistribution is the result of the incremental disorganization of the sarcomeric structures of the denervated muscle fibers, which meantime lose the normal coil distribution of subsarcolemmal myonuclei. Whatever the mechanisms of their rearrangement, the muscle nuclei are for many months (or years) regrouped into clusters of tens that fill the severely atrophic muscle fibers and are separated by long stretches of amyofibrillar myoplasm. ${ }^{23,24}$ The size of nuclear clumps is better evaluated in $1 \mu \mathrm{m}$ semi-thin longitudinally sectioned myofibers (Fig. 8). Here, stretches of amyofibrillar sarcoplasm (15-20 $\mu \mathrm{m}$ wide and 50-100 $\mu \mathrm{m}$ long) alternate with groups of tens of nuclei that fill 20-30 $\mu \mathrm{m}$ long portions of the fibers. These two $0.3 \mathrm{~mm}$ long portions of severely atrophic muscle fiber contain 10-30 myonuclei, suggesting that loss of nuclei is substantial at these late stages of muscle atrophy (in normal muscle there are 1000-2000 nuclei per mm of myofiber). ${ }^{25,26}$

These features are in sharp contrast to the stable muscle atrophy we have described in long-term paraplegics with complete upper motor neuron lesion from three to twenty years after SCI at the thoracic level. $^{27}$ Most interestingly, we have never observed nuclear clumps in that type of disuse atrophy. 


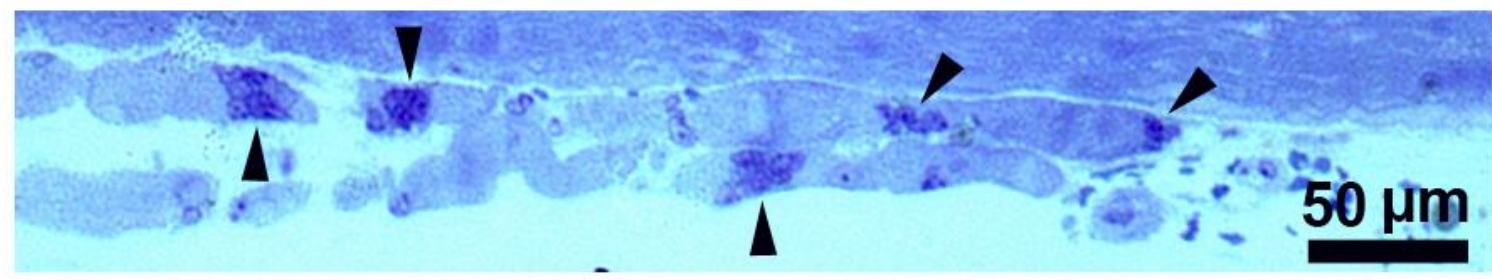

Fig 8. Four-year LMN human denervated quadriceps muscle. Longitudinal semithin section of severely atrophic myofibers. Arrowheads point to nuclear clumps that alternate with longer stretches of anucleated, amyofibrillar sarcoplasm. Toludine blue stain. Scale bar: $50 \mu \mathrm{m}$.

The only practical way to perform a quantitative analysis of nuclear clumps was to count them on muscle cross sections and then to extrapolate the data to the whole muscle. Taking into account that, after the third year of denervation, approximately $30 \%$ of atrophic myofibers show nuclear clumps, and that the clumps of nuclei are separated by longer stretches of amyofibrillar cytoplasm (Fig. 8), we calculate that almost all the severely atrophic myofibers seen in muscle biopsies from three to five years after complete, permanent LMN injury present with relocation of their nuclei from the spiral subsarcolemmal pattern of normal muscle fibers to nuclear clumps.

Up to now, nuclear groupings have been seldom described in muscle biopsies from patients affected with neurodegenerative disorders, like amyotrophic lateral sclerosis and spinal muscular atrophy. ${ }^{28}$ That is not surprising because in neurodegenerative disorders the incompleteness of denervation allows for reinnervation and thus for reorganization of larger muscle units. $^{29}$

All together, our observations confirm that human muscle fibers survive complete and permanent denervation much longer than generally accepted. Nuclear clumps should be considered markers of the long-lasting ability of human muscle fibers to survive in the absence of innervation (see Fig. $6 \mathrm{~A}$ and the following chapter). These results, together with those presented in the next two chapters, provide the rationale to plan research aimed at recovery of these severely atrophic myofibers. Specifically, we contend that further research should include the combination of molecular and cellular approaches with the functional electrical stimulation that our previous studies have shown to restore muscle structure and mass in human long-term denervated and degenerated muscle. .,18,30-40 $^{-1}$

\section{Sustained myogenesis in human long-term denervated muscles}

\section{Immunohistochemical evidence}

Muscle tissue harvested 9 months to 19 years post SCI from patients of the EU Project RISE (Contract no. QLG5-CT-2001-02191) were shown to contain the above described severely atrophic, nuclear-clumped muscle fibers (i.e., fibers surviving years of denervation) and also immature thin fibers, which are interpreted to be recently regenerating in nature. These fibers (Fig. 9A) have a central nucleus, a thin cytoplasmic rim containing a few myofilaments and also express MHCemb. ${ }^{40}$ They represent about $1 \%$ of the total myofiber population in the muscle biopsies. ${ }^{31}$ Indeed, morphologic characteristics of the long-term
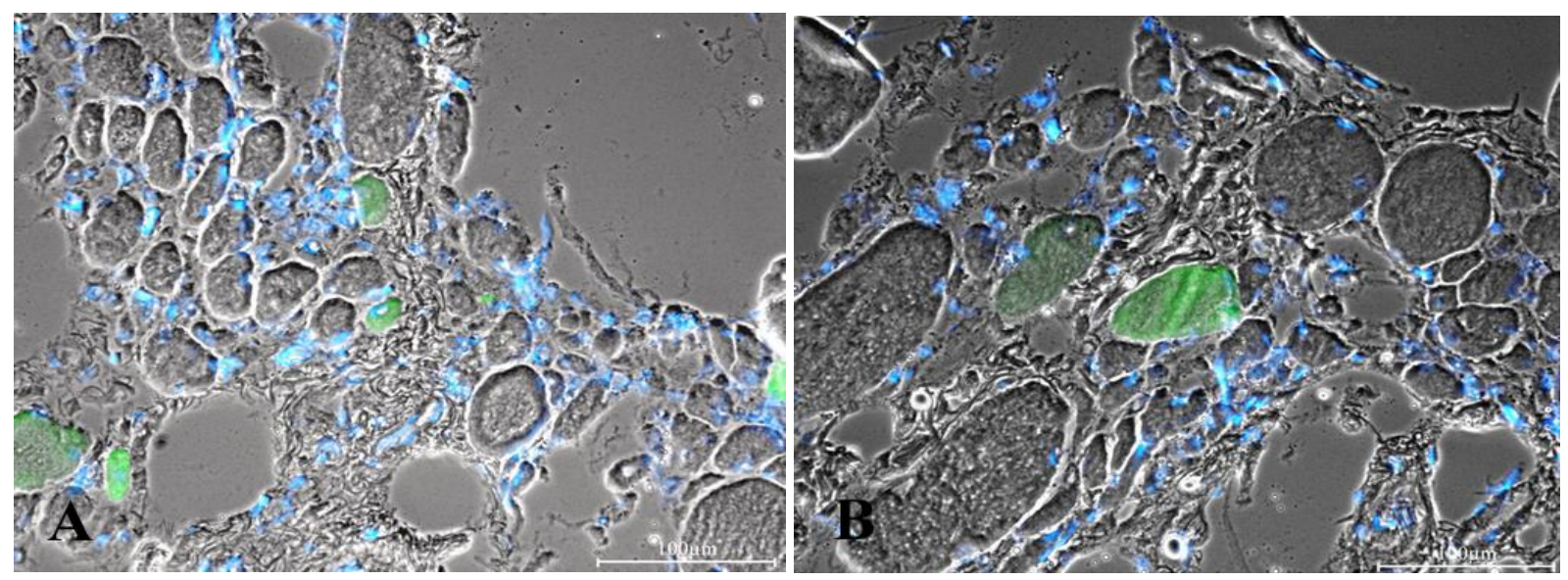

Fig 9. 2.9-year Human Denervation. MHCemb positive (green) muscle fibers are regenerated myofibers. Note in panel $A$ the centrally located small green muscle fiber presenting a central nucleus. Bar=100 $\mu \mathrm{m}$. 


\section{Muscle fiber regeneration in long term denervation}

Eur J Transl Myol - Basic Appl Myol 2015; 25 (2): 77-92

denervated muscle in animals and humans suggest that some original fibers are lost and some of those remaining are the result of repeated cycles of muscle fiber death and regeneration. Cryosections of these tissues show atrophic or severely atrophic myofibers dispersed among adipocytes and connective tissue. Monoclonal antibody for MHCemb stains 1 to $3 \%$ of the atrophic muscle fibers from 1- to 37-years postSCI. In contrast to this, after 2- to 10-years of h-bFEStraining, the muscle biopsies present with mainly large round myofibers. In the h-bFES-trained muscles the regenerative events are present, but at a lower rate than in the long-term denervated muscles not receiving $h$ bFES (MHCemb positive myofiber per $\mathrm{mm}^{2}$ of cryosection area: $0.8+/-1.3$ with h-bFES vs. $2.3+/-2.3$ vs without h-bFES biopsies, mean+/-SEM, $\mathrm{p}=0.011$ ). In our opinion, this is sound additional evidence for the positive effectiveness of the electrical stimulation protocol for h-bFES of DDM. In any case, the overall results demonstrate that h-bFES-training is safe; that is, it does not induce more myofiber necrosis followed by regeneration than denervation alone. ${ }^{31}$

In our opinion the myofibers stained by the antibody recognizing $\mathrm{MHCemb}$ had regenerated during the last few weeks before biopsy, whatever the time from SCI. This interpretation is based upon the fact that
MHCemb: 1) is expressed in myotubes and young myofibers and 2) represents the soundest molecular marker of early myogenic events in both developing and adult muscles because, in the absence of innervation, the embryonic isoforms are completely replaced by a default programm of fast-type MHC expression after two-three weeks of aneural development. ${ }^{10,41-43}$ The round, green areas seen in Fig. 9 represent cross sections of myofibers staining positively in response to anti-MHCemb antibody. They are, in our opinion, newly regenerated myofibers, which developed during the last few weeks before biopsy in the long term denervated muscles of patients suffering with complete Conus-Cauda Equina Syndrome. They are not likely to be simply reprogrammed atrophic cells. Their paucity speaks against the interpretation that they are the product of a molecular switch in myosin expression occurring in the long term denervated muscle fibers, in spite of eventual short term re-expression of embryonic protein isoforms soon after denervation. ${ }^{10}$

Doppler et al. ${ }^{44}$ found similar features in randomly chosen neurogenic muscle biopsies that did not fulfill the criterion for long-term denervation (i.e., inability to respond to electrical stimulation), because they were from patients with amyotrophic lateral sclerosis or

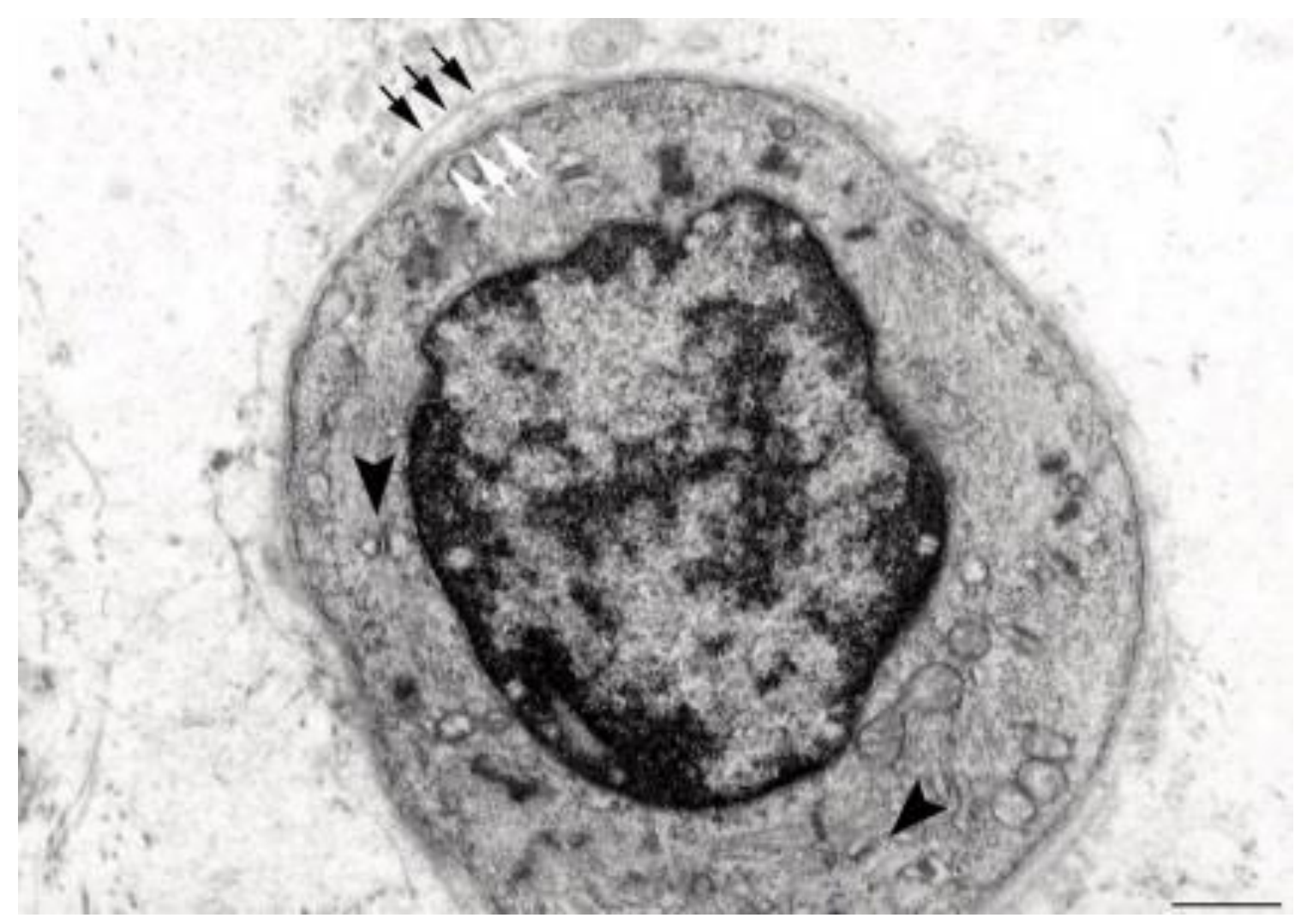

Fig 10. Electron microscopy of a transverse section of a regenerating myotube in long term (four years) denervated human muscle: two layers of basal lamina (old and new, black and white arrows, respectively) delimit a small, round fiber presenting few thick filaments, mitochondria, and triads (black arrowheads). Scale bar: $1.0 \mu \mathrm{m}$. 


\section{Muscle fiber regeneration in long term denervation}

Eur J Transl Myol - Basic Appl Myol 2015; 25 (2): 77-92

polyneuropathy. In both diseases, denervated myofibers tend to be severely atrophic and angular, resulting in an end stage of "nuclear clumps", with little cytoplasm left. ${ }^{44}$ In a cohort of 66 patients, bioptic muscle sections were immunolabeled with monoclonal antibodies against NCAM and neonatal myosin heavy

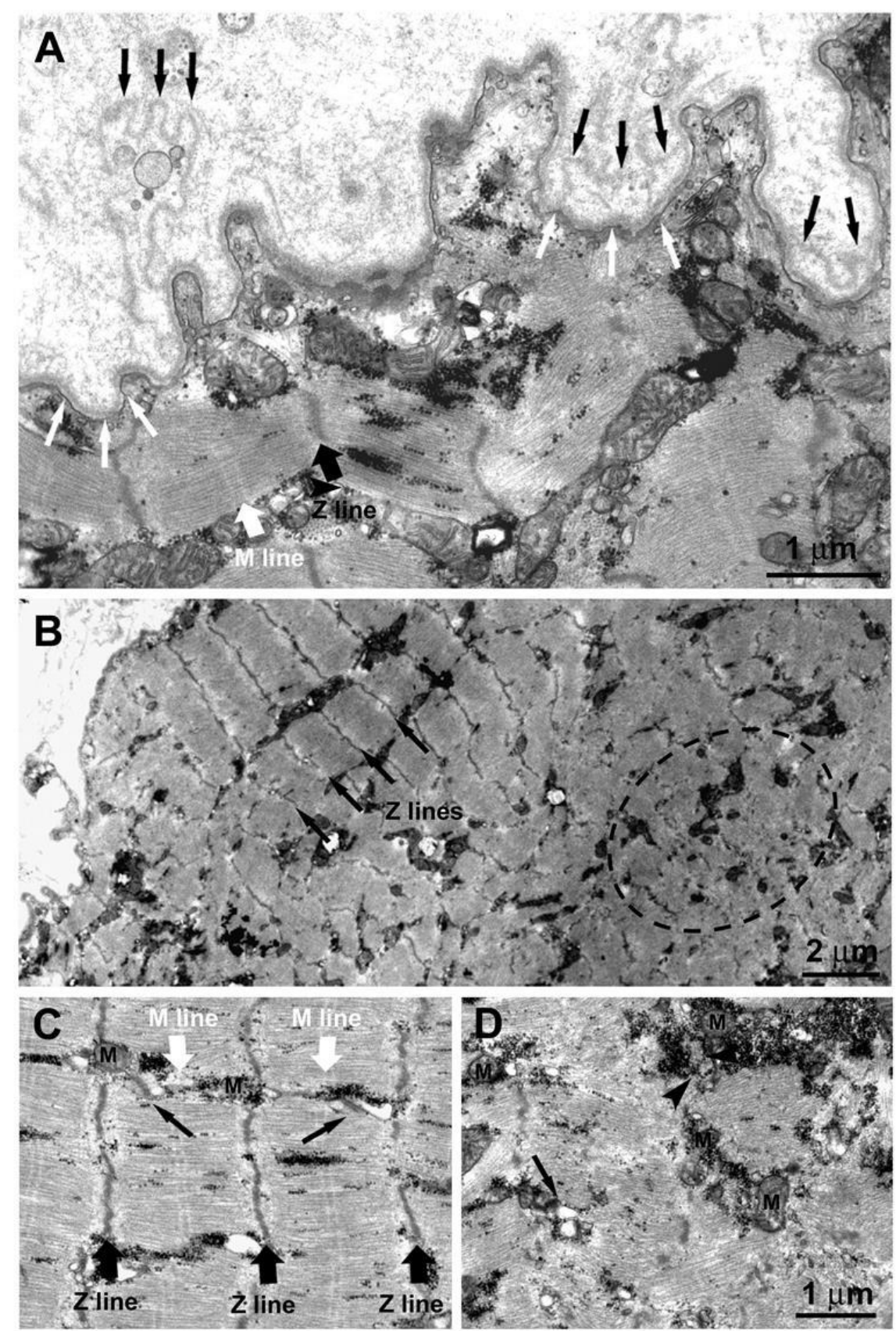

Figure 11. 1.4-year Human Denervation. Ultrastructural features of a muscle fiber undergoing atrophy due to lack of innervation. A) The double-layered basal membrane (black and white arrows) argues in favor of these being a regenerated muscle fiber. The fact that the basement membrane itself is folded (Fig. 11 A) suggests that this fiber is undergoing atrophy and degeneration due to lack of innervation. B) Within the fiber interior co-exist areas in which the sarcomeric organization of the striated muscle is slightly better preserved (black arrows pointing at the $Z$ lines) and areas presenting clear disarrangement of the contractile elements (dotted oval), more typical of muscle undergoing atrophy/degeneration. $\boldsymbol{C}$ and $\boldsymbol{D})$ Closer to the fiber periphery, where sarcomeres are better preserved with visible $Z$ and $M$ lines (black and white large arrows) the EC coupling units, normally known as triads (pointed by small black arrows), and the mitochondria $(M)$ are better positioned. On the contrary in areas in which contractile elements are degenerating, the EC coupling system is missing and/or replaced by small vesicles (arrow). Mitochondria may be found clustered, often in proximity of accumulations of glycogen granules (arrowhead). 


\section{Muscle fiber regeneration in long term denervation}

Eur J Transl Myol - Basic Appl Myol 2015; 25 (2): 77-92

chain (MHCneo), two proteins that are neurally and developmentally regulated. Of the biopsy specimens, $75 \%$ contained small myofibers that showed a thin perinuclear cytoplasmic rim. Small fibers expressing MHCneo were found in all of these biopsies (100\%) and NCAM-positive fibers were detected in $98 \%$ of the biopsies. The percentage of NCAM small fibers was significantly lower than that of MHCneo fibers. The authors conclude that myogenesis appears to be a frequent finding in human neurogenic muscle atrophy. ${ }^{44}$ Similar observations of muscle fiber regeneration were obtained in human muscle biopsies harvested from free transplanted muscle flaps that were not reinnervated. ${ }^{4}$

Our observations, thus, suggest that the MHCemb positive regenerated muscle fibers are not previous myofibers re-expressing embryonic isoforms (as is the case for acetylcholine receptors spreading from the end-plate to all the sarcolemma of recently denervated muscle fibers) $)^{3}$, but are the result of activation, proliferation and fusion of satellite cells, the myogenic precursors located under the basal lamina of the muscle fibers. ${ }^{49}$

The transient expression of laminin isoforms peculiar to early myogenesis during muscle regeneration is also well established. ${ }^{45,46}$ Further, we and others have shown that the regenerating muscle fibers, differentiating in an aneural muscle, grow up to one fourth the size of a normal adult muscle fiber, and then undergo atrophy, degeneration, and death (typically by apoptotic processes). $1,47,48$

\section{Ultrastructural evidence}

Electron microscopic analyses of muscle biopsies from the EU RISE study contribute some evidence for myofiber regeneration, i.e. the presence of doublelayered basement membranes that delimit myotubes (Fig. 10) and more developed muscle fibers (Fig. 11). We and others, ${ }^{8,45,46}$ interpreted these structures to indicate that the external membrane layer belongs to a muscle fibre that may have previously degenerated, while the internal membrane represents a new basal lamina that is being built on the surface of a regenerating myotube within the old boundary. Contrary to general expectation, but supported by rodent experiments ${ }^{1,2,6-10,13-15}$ and analyses of human muscle harvested from peripheral neuromuscular disorders, ${ }^{44}$ myofiber regeneration has been persistently observed (even if at a low rate) in longterm denervated human muscles, where presumably differentiating cells either positive to anti-MHCemb antibody or with some contractile filaments and a double layer of basal lamina are visible (Fig. 10). A few thick myofilaments with $\mathrm{Z}$ lines are present and, importantly, some triads (arrowheads in Fig. 10), that is, the $\mathrm{Ca}^{++}$-release units responsible for excitationcontraction coupling.
The thousands of muscle cultures that begin spontaneous contraction every day in myology labs worldwide are the strongest evidence that early myogenesis occurs in the absence of the nerve and its secreted factors, though they never attain full differentiation unless coinnervated. In truth, muscle fibers with a double-layered basal lamina are sparse observations in our series of denervated muscles, in agreement with the fact that anti-MHCemb antibodies stain only $1 \%$ of the myofibers in the same series of long-term denervated human muscle biopsies.

The presence of a double-layered basal membrane (Fig, 11,A, black and white arrows) argues in favor of a fiber being likely regenerated during the month before biopsy. The fiber is, however, undergoing atrophy and degeneration, presumably due to the lack of innervation as shown by extensive folding of the basement membrane. Interestingly, within the fiber interior areas with a better sarcomeric organization (Fig. 11, Panel B, black arrows pointing at the $\mathrm{Z}$ lines) coexist with others showing disarrangement of the contractile elements (Fig. 11, Panel B, dotted oval), which is more typical of muscle undergoing atrophy and degeneration. The disorganized areas (such as the one marked by the dashed oval in Fig. 11B) are usually found in the center of the fibers contrary to what happens in the long-term denervated fibers where the degeneration usually begins at the periphery. ${ }^{18,30-40}$ In addition, even in better preserved areas, myofibrils are often wavy and the sarcomeres are quite shortened. This indicates local variability in activation resulting in lack of proper alignment of contractile elements. The variability in the structural integrity of the contractile apparatus also affects the excitation-contraction (EC) coupling elements and the positioning of mitochondria, which are already quite structurally altered. Closer to the fiber periphery, where sarcomeres are better preserved with visible $\mathrm{Z}$ and $\mathrm{M}$ lines (Fig. $11 \mathrm{C}$, black and white large arrows), the EC coupling units (normally known as triads; noted by small black arrows) and the mitochondria (M) are better positioned. On the contrary, in areas where contractile elements are disarranged, the EC coupling system is missing and/or replaced by small vesicles, representing remains of sarcoplasmic reticulum (SR) membranes (Fig. 11 D, arrow). Mitochondria may be clustered, often in proximity to accumulations of glycogen granules (Fig. 11 Panel D, arrowhead).

\section{Human long term denervated muscle recovers mass and sustained contractility during the first year of $h$-bFES}

To counteract the progressive changes that transform muscle into an unexcitable tissue, over the years we have developed a novel therapeutic concept for paraplegic patients with bilateral and complete LMN denervation of the lower extremity due to complete lesion of the conus and cauda equina..$^{18,33,35,37}$ The new 


\section{Muscle fiber regeneration in long term denervation}

Eur J Transl Myol - Basic Appl Myol 2015; 25 (2): 77-92
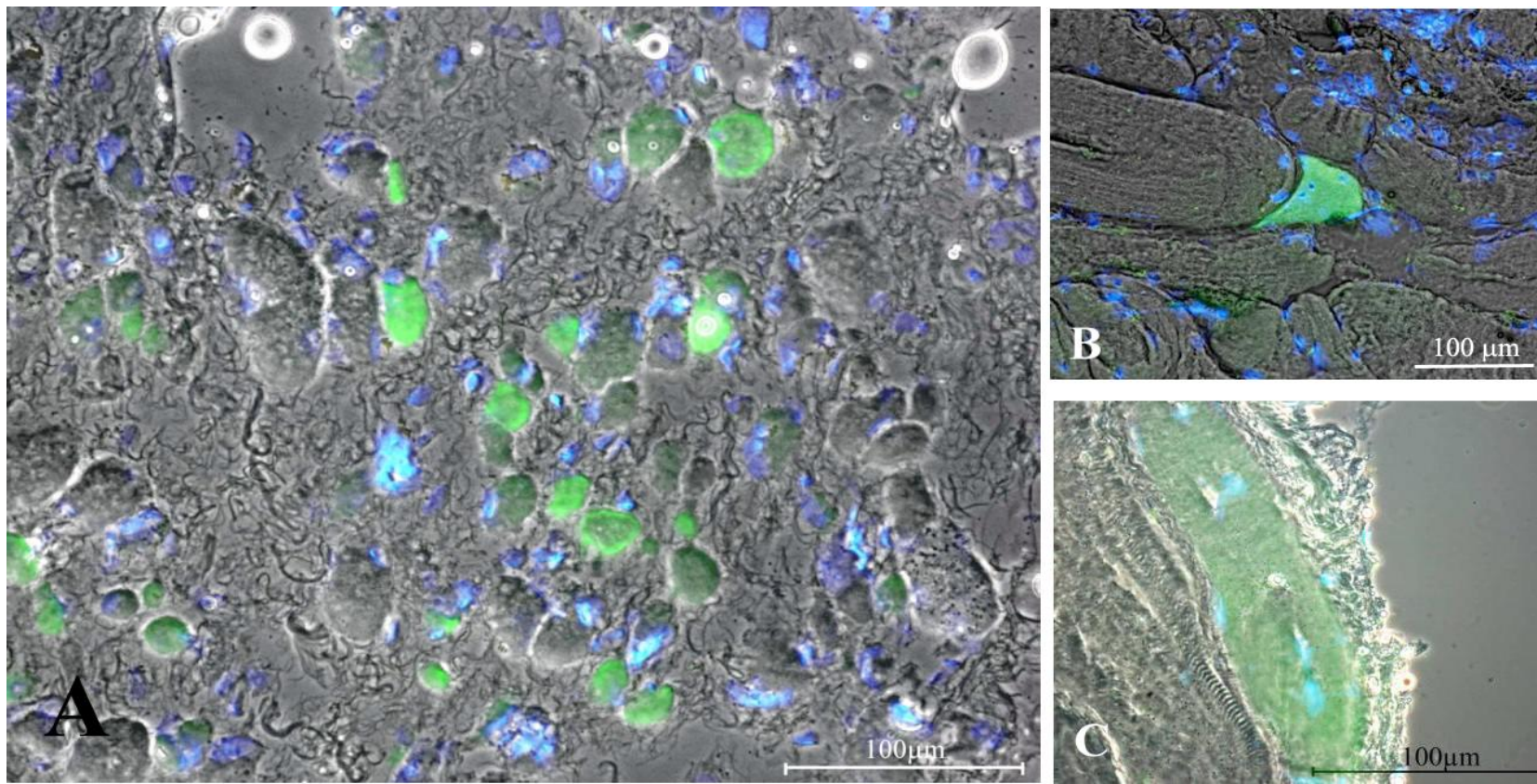

Fig. 12. Immunohistochemistry suggests the presence of regenerative events in long-term denervated human muscles without and with h-bFES. A, 3.3-year denervation without h-bFES; B, 1.2-year denervation plus additional 2.4-year of h-bFES; $C$, 1.9-year denervation plus 7.7-year h-bFES. Early regenedated myofibers stained with antibody to embryonic myosin are in green. Double exposure anti-MHCemb/Hoechst 33258 shows that some blue nuclei are centrally located. Note that in the right panels (muscle biopsies from $h$ bFES subjects, even after many more years of denervation) the myofibers are much larger than in unstimulated denervated muscles seen in A and that they have size comparable with all the other unstained muscle fibers. Scale bars: $=100 \mu \mathrm{m}$.

training strategy became possible because of the development of a new generation of stimulation equipment specifically designed for h-bFES. These new stimulators and the large surface electrodes necessary to cover the denervated muscles were developed by the Center of Biomedical Engineering and Physics at the Medical University of Vienna and by the Wilhelminenspital, Vienna (Austria). ${ }^{18,37,50-53}$ The equipment was designed to reverse long-standing and severe atrophy by delivering high-intensity and long-duration impulses that can directly elicit contraction of denervated skeletal muscle fibers, even after they have lost the ability to respond to other commercial electrical stimulators. ${ }^{18,37,50-53}$ Twenty out of 25 patients enrolled in the EU RISE trial completed the 2-year h-bFES program, which resulted in: 1) a $35 \%$ cross-sectional increase in area of the quadriceps muscle; 2) a $75 \%$ increase in mean diameter of muscle fibers; 3) improvements in the ultrastructural organization of contractile material; and 4) a $1187 \%$ increase in force output during electrical stimulation. The recovery of quadriceps force was sufficient to allow $25 \%$ of the subjects to perform FES-assisted stand-up exercises and step-training in place. ${ }^{33-37}$ It is interesting to note that in the post h-bFES muscle cryosections stained with the anti-MHCemb antibody (Fig. 12), the muscle fibers in the process of regeneration (that is, the green $\mathrm{MHCemb}$ positive muscle fibers) have the size and round aspects similar to all other muscle fibers (Fig. 12, B and C), suggesting that h-bFES stimulation allowed regenerating fibers to avoid the otherwise inevitable regeneration/atrophy cycle typical of denervated muscles. Indeed, during two years of h-bFES the fibers increased in size to levels well over that of regenerated muscle fibers differentiating into aneural muscles. ${ }^{9,10,17}$

On the basis of this evidence, we are translating these rehabilitation management strategies from long term muscle denervation cases to other cases of mobility impairments, e.g., those related to aging. ${ }^{54-59}$

\section{Conclusions and perspectives}

We have demonstrated that human denervated muscle fibers survive years of denervation and can be rescued from severe atrophy by h-bFES. We have observed the persistent presence of MHCemb positive muscle fibers (most likely regenerating muscle fibers) in both the non-stimulated and the h-bFES stimulated muscle biopsies of paraplegics with persistent, complete denervation of the quadriceps muscle. Further, we have shown that the peculiar severely atrophic fibers present in both rodent muscle (seven months after neurectomy) and in human muscle (30-months after SCI in complete Conus-Cauda Equina Syndrome) are distinguishable 


\section{Muscle fiber regeneration in long term denervation}

Eur J Transl Myol - Basic Appl Myol 2015; 25 (2): 77-92

from early myotubes using morphological features and molecular biomarkers. Beyond reviewing past evidence from rodent and human studies, here we list the options to substantially increase the regenerative potential of skeletal muscle in those patients who have experienced denervation of muscles "too-late-to-be recovered by h-bFES", that is, they present with very severely denervated muscles. Indeed, the needed mandatory procedures are ready to be translated from animal experiments to the needs of SCI persons with long-term irreversible muscle denervation, that is: 1) induction and separation of autologous myogenic cells, either i) by in vivo marcaine infiltration of an expendable muscle tissue (Latissimus Dorsi) and grown in vitro $;^{60}$ or ii) derived from autologous adipose tissue by in vitro induction and cell-sorting selection of myogenic stem cells able to replicate in vivo $;^{61-65}$ 2) multiple injections of the autologous myogenic stem cells, followed by their proliferation, fusion and differentiation into adult-like muscle fibers; and, finally, 3) their tetanic contractions induced by surface electrodes and an external neuromodulator. ${ }^{18,30-}$ $38,50-53$

Why should we not try to apply h-bFES to encourage the growth of regenerated muscle fibers, adding (to those accumulating by spontaneous activation of satellite cells) muscle fibers derived from myogenic stem cells harvested from autologous adipose tissue?

We are confident that the evidence presented here will convince ethical committees to allow animal experiments to develop the safest possible procedures for translation to those many patients who, having missed an early commencement of h-bFES, have lost muscle fibers as a consequence of the inevitable progression of denervation-induced muscle tissue degeneration that occurs in long-term Conus and Cauda Equina Syndrome and other traumatic peripheral neuropathies. Based on the evidence and concepts presented here, an European Project, the trial Rise4EU (Rise for You, a personalized treatment protocol for functional recovery of denervated muscle post long-term stable SCI), will hopefully be implemented soon.

\section{Acknowledgement}

This work was supported by European Regional Development Fund - Cross Border Cooperation Programme Slovakia - Austria 2007-2013 (InterregIVa), project Mobilität im Alter, MOBIL, N_00033 (partners: Ludwig Boltzmann Institute of Electrical Stimulation and Physical Rehabilitation, Austria, Center for Medical Physics and Biomedical Engineering, Medical University of Vienna, Austria, and Faculty of Physical Education and Sports, Comenius University in Bratislava, Slovakia); Austrian national co-financing of the Austrian Federal Ministry of Science and Research; Ludwig Boltzmann Society (Vienna, Austria); Telethon, (GGP13213) to F.P.
Some of the research reported in this publication was supported by the National Institute of Arthritis and Musculoskeletal and Skin Diseases of the National Institutes of Health under Award Number NIH NIAMS 1R03AR053706-01A2 to ALP. The content is solely the responsibility of the authors and does not necessarily represent the official views of the National Institutes of Health.

\section{Corresponding Author}

Andrea Marcante, Department of Neurorehabilitation, San Camillo Hospital, I.R.C.C.S., Venezia-Lido, Italy; and Department of Neuroscience, Rehabilitation Unit, Padua University Hospital, Padova, Italy.

E-mail: "Andrea Marcante" endriu83@gmail.com

E-mail of coauthors

Ugo Carraro: ugo.carraro@ unipd.it

Simona Boncompagni: s.boncompagni@unich.it

Valerio Gobbo: gobbov@bio.unipd.it

Sandra Zampieri: sanzamp@unipd.it

Simone Mosole: simone.mosole@studenti.unipd.it

Barbara Ravara: barbara.ravara@unipd.it

Alessandra Nori: alessandra.nori@unipd.it

Roberto Stramare: roberto.stramare@unipd.it

Francesco Ambrosio: francesco.ambrosio@unipd.it

Francesco Piccione:

francesco.piccione@ospedalesancamillo.net

Stefano Masiero: stef.masiero@unipd.it

Vincenzo Vindigni: vincenzo.vindigni@unipd.it

Paolo Gargiulo: paologar@landspitali.is

Feliciano Protasi: fprotasi@unich.it

Helmut Kern: wil.pys.kern-forschung@wienkav.at

Amber Pond: apond@siumed.edu

\section{References}

1. Borisov AB, Dedkov EI, Carlson BM. Interrelations of myogenic response, progressive atrophy of muscle fibres, and cell death in denervated skeletal muscle. Anat Rec 2001;264:203-18.

2. Carlson BM. The biology of long-term denervated skeletal muscle. Eur J Transl Myol Basic Appl Myol 2014;24:5-12.

3. Lomo T. The response of denervated muscle to long-term stimulation (1985, revisited here in 2014). Eur J Transl Myol - Basic Appl Myol 2014;24:13-9.

4. Kern H, Carraro U. Home-based Functional Electrical Stimulation (h-b FES) for long-term denervated human muscle: History, basics, results and perspectives of the Vienna Rehabilitation Strategy. Eur J Transl Myol/Basic Appl Myol 2014:24:27-40.

5. Adami N, Kern H, Mayr W, et al. Permanent denervation of rat Tibialis Anterior after bilateral sciatectomy: Determination of chronaxie by surface electrode stimulation during progression 


\section{Muscle fiber regeneration in long term denervation}

Eur J Transl Myol - Basic Appl Myol 2015; 25 (2): 77-92

of atrophy up to one year. Basic Appl Myol 2007; 17:237-434

6. Borisov AB, Dedkov EI, Carlson BM. Abortive myogenesis in denervated skeletal muscle: differentiative properties of satellite cells, their migration, and block of terminal differentiation. Anat Embryol 2005;209:269-279.

7. Schmalbruch H, al-Amood WS, Lewis DM. Morphology of long-term denervated rat soleus muscle and the effect of chronic electrical stimulation. J Physiol (Lond) 1991;441:233-241.

8. Carraro U, Morale D, Mussini I, et al. Chronic denervation of rat diaphragm: maintenance of fiber heterogeneity with associated increasing uniformity of myosin isoforms. $\mathrm{J}$ Cell Biol 1985;100:161-74.

9. Mussini I, Favaro G, Carraro U. Maturation, dystrophic changes and the continuous production of fibers in skeletal muscle regenerating in the absence of nerve. J Neurophatol Exp Neurol 1987;46:315-31.

10. Carraro U, Dalla Libera L, Catani C. Myosin light and heavy chains in muscle regenerating in absence of the nerve: transient appearance of the embryonic light chain. Exp Neurol 1983;79:10617.

11. Sartore S, Gorza L, Schiaffino S. Fetal myosin heavy chains in regenerating muscle. Nature 1982;298: 294-6.

12. Schiaffino S, Gorza L, Piton G, et al. Embryonic and neonatal myosin heavy chain in denervated and paralyzed rat skeletal muscle. Dev Biol 1988;127:1-11.

13. Carraro U, Rossini K, Zanin M, et al. Induced myogenesis in long-term permanent denervation: perspective role in Functional Electrical Stimulation of denervated legs in humans. Basic Appl Myol 2002;12:53-64.

14. Adams L, Carlson BM, Henderson L, Goldman D. Adaptation of nicotinic acetylcholine receptor, myogenin, and MRF4 gene expression to long term muscle denervation. J Cell Biol 1995;131:1341-9.

15. Lapalombella $\mathrm{R}$, Kern $\mathrm{H}$, Adami $\mathrm{N}$, et al. Persistence of regenerative myogenesis in spite of down-regulation of activity-dependent genes in long-term denervated rat muscle. Neurol Res 2008;30:197-

16. Squecco R1, Carraro U, Kern H, Pond A, et al. A subpopulation of rat muscle fibers maintains an assessable excitation-contraction coupling mechanism after long-standing denervation despite lost contractility. J Neuropathol Exp Neurol. 2009 Dec;68(12):1256-68. doi: 10.1097/NEN.0b013e3181c18416.

17. Carlson BM. "The Denervated Muscle" - 45 years later. Basic Appl Myol 2007;17:113-7.
18. Kern H, Boncompagni S, Rossini K, et al. Longterm denervation in humans causes degeneration of both contractile and excitation contraction coupling apparatus, wich is reversible by functional electrical stimulation (FES). A role for myofiber regeneration? J Neuropathol Exp Neurol 2004;63:919-31.

19. Biral D, Kern H, Adami N, et al. Atrophyresistant fibers in permanent peripheral denervation of human skeletal muscle. Neurol Res 2008;30:137-44.

20. Ashley Z, Sutherland H, Lanmuller $H$, et al. Atrophy, but not necrosis, in rabbit skeletal muscle denervated for periods up to one year. Am J Cell Physiol 2007;292:440-51.

21. Lewis DM, Al-Amood WS, Schmalbruch H. Effects of long-term phasic electrical stimulation of denervated soleus muscle: guinea pig contrasted with rat. J Muscle Res Cell Motil 1997; 18:573-86

22. Carraro U, Catani C, Saggin L, et al. Isomyosin changes after functional electrostimulation of denervated sheep muscle. Muscle Nerve. 1988;11:1016-28.

23. Kern H, Carraro U, Biral D, et al. Severely atrophic muscle fibers with nuclear clumps survive many years in permanently denervated human muscle. The Open Pathology Journal 2009;3:106-10.

24. Adami N, Biral D, Carraro $U$ et al. The fiber types of severely atrophic muscle fibers with nuclear clumps of human 5-year LMN denervated muscle. Basic Appl Myol 2009;19:225-8.

25. Aravamudan B, Mantilla CB, Zhan WZ, Sieck GC. Denervation effects on myonuclear domain size of rat diaphragm fibers. J Appl Physiol 2006;100:1618-22.

26. Gundersen K, Bruusgaard JC. Nuclear domains during muscle atrophy: nuclei lost or lost paradigm. J Physiol 2008;586:2675-81.

27. Kern H, Hofer C, Modlin M, et al. Stable muscle atrophy in long term paraplegics with complete upper motor neuron lesion from 3- to 20-year SCI. Spinal Cord 2008;46:293-304.

28. Dubowitz V, Sewry CA. Muscle biopsy. A practical approach. Sauders Elsevier Edition 2007. Neurol 2004;63:919-31

29. Wokke JH. Genes, trials, and care: The dynamics of neuromuscular disease. Lancet Neurol 2004;3:1-16.

30. Kern H, Hofer C, Mayr W, Carraro U. European Project RISE: Partners, protocols, demography. Basic Appl Myol/Eur J Transl Myol 2009;19:2116.

31. Carraro U, Rossini K, Mayr W, Kern H. Muscle fiber regeneration in human permanent lower motoneuron denervation: relevance to safety and effectiveness of FES-training, which induces 


\section{Muscle fiber regeneration in long term denervation}

Eur J Transl Myol - Basic Appl Myol 2015; 25 (2): 77-92

muscle recovery in SCI subjects. Artif Organs 2005;19:187-191.

32. Kern H, Salmons S, Mayr W, et al. Recovery of long-term denervated human muscles induced by electrical stimulation. Muscle Nerve 2005;31:98101.

33. Boncompagni $\mathrm{S}$, Kern $\mathrm{H}$, Rossini $\mathrm{K}$, et al. Structural differentiation of skeletal muscle fibers in the absence of innervation in humans. Proc Natl Acad Sci USA 2007;104:19339-44.

34. Kern H, Carraro U. Translational myology focus on clinical Challenges of functional electrical stimulation of denervated muscle. Basic Appl Myol/Eur J Transl Myol 2008;18:37-100.

35. Kern H, Carraro U, Adami N, et al. One year of home-based Functional Electrical Stimulation (FES) in complete lower motor neuron paraplegia: Recovery of tetanic contractility drives the structural improvements of denervated muscle. Neurol Res 2010;32:5-12, doi: $10.1189 / 184313209$ X385644.

36. Kern H, Hofer C, Mayr W. Protocols for clinical work package of the European project RISE. Basic Appl Myol/Eur J Transl Myol 2008;18:3944.

37. Kern H, Carraro U, Adami N, et al. Home-based functional electrical stimulation rescues permanently denervated muscles in paraplegic patients with complete lower motor neuron lesion. Neurorehabil Neural Repair. 2010;24:70921. Epub 2010 May 11.

38. Gargiulo P, Helgason T, Reynisson PJ, et al. Monitoring of muscle and bone recovery in spinal cord injury patients treated with electrical stimulation using three-dimensional imaging and segmentation techniques: methodological assessment. Artif Organs 2011;35:275-81. doi: 10.1111/j.1525-1594.2011.01214.x.

39. Mancinelli R, Kern H, Fulle $S$, et al. Transcriptional profile of denervated vastus lateralis muscle derived from a patient 8 months after spinal cord injury: a case-report. Int $\mathbf{J}$ Immunopathol Pharmacol 2011;24:749-59.

40. Rossini K, Zanin ME, Carraro U. To stage and quantify regenerative myogenesis in human longterm permanent denervated muscle. Basic Appl Myol 2002;12;277-87.

41. Whalen RG, Sell SM, Butler-Browne GS, et al Three myosin heavy-chain isozymes appear sequentially in rat muscle development. Nature 1981;292:805-9.

42. Lewis DM, Schmalbruch H. Contractile properties of aneurally regenerated compared with denervated muscles of rat. J Muscle Res Cell Motil 1994;15:267-77.

43. Carraro U, Catani C. A sensitive SDS PAGE method separating heavy chain isoforms of rat skeletal muscles reveals the heterogeneous nature of the embryonic myosin. Biochem Biophys Res Commun 1983;116:793-802.

44. Doppler K, Mittelbronn M, Bornemann A. Myogenesis in human denervated muscle biopsies. Muscle Nerve 2008;37:79-83.

45. Gulati AK. Basement membrane component changes in skeletal muscle transplants undergoing regeneration or rejection. $\mathrm{J}$ Cell Biochem 1985;27:337-46.

46. Grounds MD, McGeachie JK, Davies MJ, et al. The expression of extracellular matrix during adult skeletal muscle regeneration: how the basement membrane, interstitium and myogenic cells collaborate. Basic Appl Myol 1998;8:129141.

47. Sandri M, Carraro U, Podhorska-Okolov et al. Apoptosis, DNA damage and ubiquitin expression in normal and mdx muscle fibers after exercise. FEBS Lett 1995;373:291-5.

48. Carraro U, Franceschi C. Apoptosis of skeletal and cardiac muscles and physical exercise. Aging (Milano). 1997;9:19-34. Review.

49. Yablonka-Reuveni $Z$. The skeletal muscle satellite cell: still young and fascinating at 50. J Histochem Cytochem 2011;59:1041-59. doi: 10.1369/0022155411426780.

50. Hofer C, Mayr W, Stöhr H, et al. A stimulator for functional activation of denervated muscles. Artif Organs 2002;26:276-9.

51. Mayr W, Bijak M, Rafolt D,et al. Basic design and construction of the Vienna FES implants: existing solutions and prospects for new generations of implants. Med Eng Phys 2001;23:53-60.

52. Mödlin M, Forstner C, Hofer C, et al. Electrical stimulation of denervated muscles: first results of a clinical study. Artif Organs 2005;29:203-6.

53. Gargiulo P, Reynisson PJ, Helgason B, et al. Muscle, tendons, and bone: structural changes during denervation and FES treatment. Neurol Res 2011;33:750-8. doi: 10.1179/1743132811 Y.0000000007.

54. Kern H, Barberi L, Löfler S, et al. Electrical stimulation counteracts muscle decline in seniors. Front Aging Neurosci. 2014 Jul 24;6:189. doi: 10.3389/fnagi.2014.00189. eCollection 2014.

55. Mosole S, Carraro U, Kern H, et al. Long-term high-level exercise promotes muscle reinnervation with age. J Neuropathol Exp Neurol 2014;73:284-94. doi: 10.1097/NEN.00000000000 00032 .

56. Zampieri S, Pietrangelo L, Loefler $\mathrm{S}$, et al. Lifelong physical exercise delays age-associated skeletal muscle decline. J Gerontol A Biol Sci Med Sci 2014 Feb 18. [Epub ahead of print]

57. Marcante A, Zanato R, Ferrero M, et al. Recovery of tetanic contractility of denervated muscle: A step toward a walking aid for foot drop. Biomed 


\section{Muscle fiber regeneration in long term denervation}

Eur J Transl Myol - Basic Appl Myol 2015; 25 (2): 77-92

Tech (Berl) 2013 Sep 7. pii: /j/bmte.2013.58.issue-s1-A/bmt-2013-4016/bmt2013-4016.xml. doi: 10.1515/bmt-2013-4016. [Epub ahead of print]

58. Zanato R, Stramare R, Boato N, et al. Dynamic echomyography shows that FES in Peripheral Denervation does not hamper muscle reinnervation. Biomed Tech (Berl) 2013 Sep 7. pii:/j/bmte.2013.58.issue-s1-A/bmt-20134034/bmt-2013-4034.xml. doi: 10.1515/bmt2013-4034. [Epub ahead of print].

59. Kern H, Pelosi L, Coletto L, et al. Atrophy/ hypertrophy cell signaling in muscles of young athletes trained with vibrational-proprioceptive stimulation. Neurol Res 2011;33:998-1009. doi: 10.1179/016164110X127 67786356633.

60. Péault B, Rudnicki M, Torrente Y, et al. Stem and Progenitor Cells in Skeletal Muscle Development, Maintenance, and Therapy. Mol Ther 2007;15:867-77. Epub 2007 Mar 27. doi: 10.1038 /mt.sj.6300145.

61. Lindroos B, Suuronen R, Miettinen S. The potential of adipose stem cells in regenerative medicine. Stem Cell Rev 2011;7:269-91. doi: 10.1007/s12015-010-9193-7.
62. Zuk PA, Zhu M, Ashjian P, et al. Human adipose tissue is a source of multipotent stem cells. Mol Biol Cell 2002;13:4279-95.

63. Russo V, Yu C, Belliveau P, et al. Comparison of human adipose-derived stem cells isolated from subcutaneous, omental, and intrathoracic adipose tissue depots for regenerative applications. Stem Cells Transl Med 2014;3:206-17. doi: 10.5966/sctm.2013-0125. Epub 2013 Dec 20.

64. Kern H, Carraro U. Home-based Functional Electrical Stimulation for long-term denervated human muscle: History, basics, results and perspectives of the Vienna Rehabilitation Strategy. Eur J Transl Myol - Basic Appl Myol 2014;24:27-40. doi: 10.4081/bam.2014.1.27

65. Huang H, Sun T, Chen L, et al. Consensus of clinical neurorestorative progresses in patients with complete chronic spinal cord injury. Cell Transplant 2014;23 Suppl 1:5-17. doi: 10.3727/096368914X684952. Epub 2014 Oct 9.

66. Adami N, Biral D, Corbianco S, et al. The fiber types of severely atrophic muscle fibers with nuclear clumps of human 5-year LMN denervated muscle. Basic Appl Myol 2009;19:225-8. 